\title{
The individual (single patient) IND for inborn errors of metabolism
}

Patricia I. Dickson ${ }^{1, *}$ and Jakub Tolar ${ }^{2}$

1. Dept. of Pediatrics, Los Angeles Biomedical Research Institute at Harbor-UCLA Medical Center, Torrance, CA

2. Dept. of Pediatrics and Stem Cell Institute, University of Minnesota, Minneapolis, MN

* Corresponding author: Patricia I. Dickson, M.D.

1124 W. Carson Street, HH1

Torrance, CA 90502

USA

Tel: $\quad 310-781-1399$

Fax: $\quad 310-781-1093$

Email: pdickson@ucla.edu

Formatted for Molecular Genetics and Metabolism (commentary) 


\begin{abstract}
Under the United States Food and Drug Administration's Expanded Access program, a physician may treat a single patient with an experimental medication under an individual investigational new drug application (iIND). Metabolic geneticists may be among the specialists most likely to be asked to obtain an iIND, because there are many experimental treatments for inborn errors of metabolism which work in animals but suffer delays in translation to the bedside. The iIND has the potential to help bridge that gap, by gathering initial evidence in support of the use of an experimental drug in humans. If done with experimental rigor, the data will be useful, despite being limited to a single patient. However, iINDs may pose risks to drug development if patients are not carefully selected. Whatever their advantages and disadvantages, iIND studies are here to stay. Metabolic specialists should cautiously consider the iIND as a tremendous opportunity for therapeutic experimentation.
\end{abstract}




\section{Introduction}

There are no available treatments for the vast majority of rare disorders including many inborn errors of metabolism. Yet, there are many experimental treatments which have shown success in animal models, but have not reached the clinic due in part to challenges in translation of therapies for rare diseases [1,2]. Expanded access allows a physician to request an experimental therapy for the treatment of a single patient [3]. Expanded access is limited to patients with life-threatening conditions and no effective treatment options. The most commonly used mechanism for this effort is the single patient or individual investigational new drug application (iIND). For example, the iIND has been used by several families affected by Niemann-Pick type C disease in order to gain access to 2-hydroxypropyl-beta-cyclodextrin, before phase I human investigations were conducted for that indication [4]. The increasing public attention to iINDs has led to concerns of some within the scientific community that the iIND studies will interfere with ongoing clinical trials. The most obvious problem is that iIND studies might diminish the pool of available subjects from which to recruit. This can be a serious matter for very low-prevalence diseases including many of the inborn errors of metabolism. Another concern is that iIND studies could introduce additional risk both to patient safety and to eventual drug approval, because iIND studies cannot exercise the rigorous control over patient selection and study procedures that occur in a clinical trial to minimize the possibility of serious adverse events.

However, there are reasons that physicians and families may seek to use the iIND mechanism. Clinical trials are difficult and costly. For very rare disorders such as inborn errors of metabolism, there are added delays due to difficulties in attracting commercial interest, lengthy natural history studies that may be needed in order to select primary outcome measures, and a limited population of patients from which to enroll into a trial. While the iIND was designed for the treatment of patients, in practice the iIND permits early human experience with 
a potential therapy which can help guide this process. In addition, the iIND may have uses beyond complementing traditional drug development. The iIND also presents an opportunity to practice true personalized medicine.

\section{What is an IND, and what is an iIND?}

An iIND is an IND submitted for the experimental treatment of a single patient. The iIND mechanism is limited to patients suffering from a life-threatening disorder without available effective treatment. Pariser and colleagues provide further information on the iIND in this issue of the journal [5].

\section{Potential benefits to science}

The intent of the iIND is to provide access to potentially life-saving experimental drugs to patients who could not otherwise get them. However, there may be unforeseen and unique benefits to science and to a drug's development program from a carefully designed and conducted iIND study. The potential benefits that we outline here represent our own opinions and reflections. They are based on our experience with the design and conduct of phase 1 clinical trials and iIND studies for rare diseases. There is not evidence from the literature, or of which we are otherwise aware, to support the role of the iIND in successful drug registration. Before going further to explain our thoughts on the potential benefits of iINDs, it is necessary to first explain some of the challenges posed by traditional phase 1 studies.

3.1 The opportunity to gain early human experience using an iIND. Phase 1 studies represent first in human testing and are designed to detect frequent adverse events and often to collect pharmacokinetic data. As a phase 1 study is meant to address safety and pharmacology, efficacy is not theoretically expected. However, the reality is that due to the large investment that is 
made by drug developers, coupled with the expectations of patients and families, if a drug fails to show any efficacy in phase 1 there may be no motivation nor practical way to further expand the study in the particular disease population. One reason is that the internet enables families to communicate their experiences instantaneously, and if a drug shows no impact on disease in initial testing, a sponsor will find great difficulty recruiting subjects for the next phase of development. Many drug sponsors circumvent this problem by combining phase 1 and 2 (phase 1/2 studies), and therefore they collect initial efficacy data by design. The drawback to phase $1 / 2$ studies is that there is little room for adaptive approach to relevant biomedical discovery - the investigator or sponsor must have included any endpoints that they believe may show improvement and they must have decided prior to clinical experimentation what frequency and mode of administration to utilize before the study begins. The price for failure may be another costly study, or - more typically - the end to a drug development program altogether.

An iIND may offer some advantages to a physician who is seeking to study the use of an experimental therapy in his or her patient (Table 1). Although an iIND protocol is typically not written to collect data in a rigorous fashion, there is no a priori reason that it cannot be so, and the motivation of the primary investigator is to learn from the iIND intervention as much as is possible. If an iIND study is designed and conducted rigorously, it will provide first-in-human data and reveal practical information about treating a patient population before expensive trials begin.

3.2. The iIND for experimental therapies under active clinical investigation. The role of the iIND in providing useful data is less obvious for clinical interventions that are currently under active clinical investigation. However, even in a situation where phase 1 investigations are already underway, an iIND can be used under certain circumstances. Studying a single patient allows flexibility, and enables the physician to adapt the treatment to the needs of the patient in a way that the more rigid clinical study protocol may not permit. Adapting the treatment to the 
specifics of the patient could in theory improve the chances of detecting an efficacy signal, or conversely, decrease the risk of adverse events. The iIND study could also be designed slightly differently to the clinical trial, in order to provide additional data. For example, the iIND study could evaluate a different dose, dosing frequency or mode of administration, or it could include the investigation of outcome measures that were not included in the phase 1 study protocol. To maximize the potential for iIND studies to provide high-quality data for a drug development program, it is helpful if physicians follow a similar protocol, exchange information, and collaborate with ongoing research. As a current example, several active iIND studies of 2hydroxypropyl-beta-cyclodextrin for Niemann-Pick C disease follow a modified version of the protocol for the phase 1 study at the National Institutes of Health, and send samples to the same laboratory for biomarker evaluation.

3.3. Personalized medicine using the iIND. The iIND may also be a means by which we can begin to contemplate personalized medicine. The concept of personalized medicine bridges both rare and common disorders. Technologies such as stop-codon read-through and gene repair may be mutation-specific. When a mutation-specific therapy is applied to a rare disease, the pool of recruitable subjects becomes very small indeed. Also, as we use, analyze, and organize the wealth of genetic and pharmacokinetic data currently available, we will be able to tailor even common drugs more specifically to each individual. Thus, it is conceivable - even likely - that in both rare and common disorders we will see a departure from massive clinical trials in favor of small or even of " $\mathrm{N}$ of 1 " trials that are rationally tailored to actionable biomedical information on personal level.

\section{Practical issues and problems with iIND studies}

There is controversy as to whether it is desirable to use an iIND to provide access to a drug that is under active development. The two major problems with iINDs in this situation are 
1) they reduce the pool of potential subjects for recruitment into active clinical trials (in which case, an iIND should not be granted), and 2) they may introduce risk both to the patients receiving the drug and to the drug development program. Expanded access is allowed only in life-threatening situations, in which risk to the individual patient is low compared to the certain outcome of disease progression. Risk to the drug-development program comes about because iIND studies do not generally involve rigorous patient selection. For example, an iIND may be granted to a patient in the terminal stages of a rare disorder, as there are no other possible treatment options available. However, terminally-ill patients may have an increased risk of experiencing drug-related adverse events, due to poor overall health. Viewed differently though, should the medication prove useful in extremis it may reveal a mechanism of action that may be undetectable in earlier and milder manifestations of the same disease. Also, the risk of experimental therapy in terminally-ill patients it is perceived by the patient's family and as a real life bedside experience is incomparable to such risk in less serious circumstances: for individuals with potentially fatal disorders just living with that disorder is risky and they will see the risks of novel therapy relative to that risk. From the standpoint of the sponsor the drugrelated adverse events, however, may jeopardize the drug development program. In this way, the use of iINDs may retard the progress of drugs through the clinical trial stages.

Physician-investigators who hold an iIND for a patient may experience a number of practical issues with carrying these studies out. First, medical insurance may or may not cover the costs related to the treatment of the patient under an iIND. Depending on the protocol and route of administration, these can be extremely high, particularly if hospitalization is needed for dosing or monitoring. Pre-authorization is time-consuming, and even when this process is followed the patient may receive a bill for services. In addition to costs related to the experimental treatment, there are often additional costs to the conduct of iIND studies. These include IRB fees, shipping and storage of patient samples, investigational drug service costs, and services of a study coordinator. Some of these expenses may individually be small, but 
collectively are difficult to manage as there is no grant account that the institution can bill for the service. Finally, the time commitment is substantial, including IRB and regulatory forms and approvals, communication and consent (an ongoing process), care of the patient, charting, data collection and management, and data reporting. Both the time commitment and nonbillable costs increase with the experimental rigor of the study protocol. In other words, if the physician-investigator wants to collect more valuable experimental data from the iIND study, he or she will need to invest more time and find ways to cover the increased costs associated with the study procedures and data. In any case, collecting reimbursement for an iIND study requires the physician holding the iIND to file a "request to charge," in which a detailed list of all expenses is provided and certified by an independent accountant.

A physician who desires to use an iIND to collect data about the safety and efficacy of an experimental therapy in a patient may find himself or herself in conflict with the desires of the family to have "treatment" for the patient's disease. The "therapeutic misconception," or the conflation of an experimental therapy with a "proven treatment" is a common problem in clinical trials and applies to iIND studies as well. In the case of an iIND, there is only one patient, and the family may view the protocol as being specifically tailored to that patient's needs. This arrangement may lead to expectations by the patient and family that they will have control over the conduct of the iIND investigation. The family will need careful counseling about the experimental nature of the iIND protocol before, during, and after the informed consent process. The physician leading the study should know how he or she will handle the patient and family's demands for protocol flexibility, and discuss the family's expectations in this regard. This conversation is especially important in the case where the iIND is structured to provide meaningful experimental data.

\section{Physicians on the front line}


The FDA has a mandate to provide expanded access to experimental drugs and treatments under appropriate circumstances. A growing awareness of this option means that more and more patients and their families will seek iINDs. The bulk of iIND requests that a metabolic specialist receives may come from families of patients with rare, incurable and lifethreatening diseases for which there may be experimental treatments that are "stuck" in the valley between basic science discovery and clinical translation. With more volume and velocity of clinically meaningful insights from better understanding of mechanisms of genetic diseases, physicians caring for patients with rare diseases are increasingly going to be on the front lines, having to decide whether to pursue or refuse a family's request for iIND treatment. If placed in this situation, we suggest that the physician do the following before deciding whether to proceed:

- Master the relevant literature and seek counsel from investigators who are knowledgeable about the experimental use of the drug. Try to find out how likely the treatment is to help or harm your patient.

- If there is an active clinical trial, talk to the principal investigator of that trial to make sure that enrolling your patient in an iIND will not conflict with the conduct of the trial or to future planned trials along the drug development pathway.

- Write a study protocol that will collect objective, quantitative data on the safety and effectiveness of the experimental therapy in your patient. If possible, follow a common study protocol in order to maximize the chances that the data you collect will be interpretable in the context of other iINDs and/or clinical trials.

- Make sure that you understand what the patient and their family expects, and make sure that they understand what to expect from you. For example, the family must understand whether you need them to adhere strictly to a study protocol including schedule, reporting adverse events, and permitting procedures aimed at data collection. 
The iIND is not designed for research, and phase I clinical trials are the standard in any drug development program. The National Institutes of Health and the Food and Drug Administration offer several funding mechanisms, including NeuroNEXT, Rare Disease Clinical Research Networks, and the Orphan Products Development grant program. These programs support clinical trials for novel therapies in patients with inborn errors of metabolism. Additional funding mechanisms for small clinical trials, including $n=1$ studies for extremely rare diseases or patient-tailored therapeutics, are needed in order to bring the latest therapeutic options to patients.

\section{Conclusions:}

An iIND provides experimental therapy to a patient with a life threatening condition and no alternative treatments for their disease. If carried out with experimental rigor, an iIND could provide valuable data in support of safety and efficacy of an experimental treatment. However, iIND studies done incorrectly or in the wrong circumstances may pose a risk to a patient and to a drug development program. The iIND thus presents both a challenge and an opportunity to generate pilot evidence that would otherwise take decades to gain or may not be accessible by the current format of clinical trials at all. Physicians should proceed with caution, but ultimately should view the iIND as a way to align discoveries with adaptable clinical research in order to bring novel and meaningful benefits to their patients.

\section{Disclosures}

Patricia Dickson receives research support from BioMarin, Shire, Genzyme-Sanofi, Isis Pharmaceuticals, and Armagen Pharmaceuticals.

\section{Acknowledgements}


Supported in part by NIH/NINDS Ro1NSo85381 to P.I.D. and NIH/NCATS (UCLA CTSI)

UL1TRoo0124. JT is supported in part by grants from the National Institutes of Health (Ro1

ARo63070 and Ro1AR059947), US Department of Defense (W81XWH-12-1-0609),

Epidermolysis Bullosa Research Fund, Jackson Gabriel Silver Foundation, and DebRA.

\section{References:}

[1] P.I. Dickson, A.R. Pariser, S.C. Groft, R.W. Ishihara, D.E. McNeil, D. Tagle, et al., Research challenges in central nervous system manifestations of inborn errors of metabolism, Mol. Genet. Metab. 102 (2011) 326-338. doi:10.1016/j.ymgme.2010.11.164.

[2] J. Vockley, C.M.W. Vockley, Clinical trials: Curing a critical deficiency in metabolic medicine, Mol. Genet. Metab. 99 (2010) 244-245. doi:10.1016/j.ymgme.2009.12.001.

[3] Expanded access to investigational drugs for treatment use. Final rule., Fed. Regist. 74 (2009) 40900-45.

[4] A.D. Marcus, TRIALS: A Fight for Children with Niemann-Pick Type C, a Fatal Genetic Disease, Wall Str. J. (2014).

[5] A.R. Pariser, TITLE TBA, Mol Genet Metab. oo (n.d.) ooo-ooo. 
Table 1: Advantages and disadvantages of iIND studies for rare disease treatments

\begin{tabular}{|l|l|}
\hline Advantages of iIND studies & $\begin{array}{l}\text { Disadvantages and practical problems } \\
\text { of iIND studies }\end{array}$ \\
\hline $\begin{array}{l}\text { Provides access to experimental treatment to } \\
\text { patients }\end{array}$ & $\begin{array}{l}\text { Loose control over study conduct and data } \\
\text { collection }\end{array}$ \\
\hline $\begin{array}{l}\text { Low-risk collection of safety information (one } \\
\text { subject placed at risk) }\end{array}$ & $\begin{array}{l}\text { Potential risk to drug development program } \\
\text { from poor patient selection }\end{array}$ \\
\hline $\begin{array}{l}\text { Flexible, can adapt treatment to needs of } \\
\text { patient }\end{array}$ & $\begin{array}{l}\text { Physician pressure and expectations of } \\
\text { patients and families }\end{array}$ \\
\hline $\begin{array}{l}\text { Early potential problems in study design, } \\
\text { drug delivery, etc. can be identified }\end{array}$ & $\begin{array}{l}\text { No or inadequate reimbursement for time } \\
\text { and costs from iIND studies }\end{array}$ \\
\hline $\begin{array}{l}\text { If a similar protocol is used, data may be } \\
\text { roughly comparable across iIND studies }\end{array}$ & $\begin{array}{l}\text { Data from multiple iIND studies cannot be } \\
\text { formally compared }\end{array}$ \\
\hline
\end{tabular}

